Archives de sciences sociales des religions

116 | octobre - décembre 2001

Varia

\title{
En finir avec le bricolage ... ?
}

André Mary

\section{OpenEdition}

Journals

Édition électronique

URL : http://journals.openedition.org/assr/494

DOI : $10.4000 /$ assr.494

ISSN : $1777-5825$

Éditeur

Éditions de l'EHESS

Édition imprimée

Date de publication : 2 octobre 2001

Pagination : 27-30

ISBN : 2-222-96712-0

ISSN : 0335-5985

Référence électronique

André Mary, «En finir avec le bricolage ... ? », Archives de sciences sociales des religions [En ligne],

116 | octobre - décembre 2001, mis en ligne le 12 octobre 2005, consulté le 21 avril 2019. URL : http:// journals.openedition.org/assr/494; DOI : 10.4000/assr.494 


\section{EN FINIR AVEC LE BRICOLAGE ...?}

Dans une formule relativement nouvelle, la revue des Archives a bien voulu se faire l'écho du débat que l'AFSR a organisé en décembre 2000 autour de mon livre sur Le bricolage africain des héros chrétiens (cf. Arch. 114, pp. 39-59). Ce débat a été organisé par Françoise Champion dans l'esprit d'une rencontre entre anthropologues et sociologues et d'une confrontation sur la pertinence de la notion de bricolage au regard des productions de sens que chacun est appelé à analyser dans différents contextes, néo-traditionnels ou postmodernes, européens, africains ou afro-américains. Je m'autorise ici à revenir en quelques mots sur les termes de ce débat, non pas pour me livrer à un quelconque droit de réponse, mais pour compléter la publication des interventions des uns et des autres par le résumé de quelques réflexions qu'elles ont suscitées de ma part aussi bien lors du débat qu'à la relecture. Car cet échange sur les usages « relativement libres»-les mésinterprétations ou les « ré-emplois »-que sociologues et anthropologues font du concept ou de la métaphore du «bricolage », s'est révélé particulièrement fécond, en prise directe sur les divergences qui peuvent exister dans l'analyse de phénomènes religieux contemporains, et me conduit personnellement à clarifier certaines thèses sur le «bris-collage » dans sa version post-moderne ou à lever quelques malentendus.

Faut-il dire et redire que mon intérêt pour les syncrétismes n'a jamais été lié au souci de typologiser des configurations religieuses propres à une aire culturelle ou à une situation historique donnée en les qualifiant de « syncrétiques » ? Mon travail n'est pas un travail de théologien. Il s'agit de cerner, par le biais notamment de l'investissement ethnographique de certains dispositifs rituels, les modalités d'un certain type de travail symbolique particulièrement présent au cœur de certaines productions ou entreprises religieuses. On peut parfaitement soutenir qu'il faut en finir avec une catégorie cléricale héritée de l'histoire missionnaire ou avec les faux problèmes qu'engendre l'idée d'une religion pure, chrétienne ou païenne : le syncrétisme est partout et nulle part. Mais qu'on se propose de faire table rase de la notion ou d'aller " au-delà du syncrétisme », il faut bien admettre que : " chasser le syncrétisme, il revient au galop ». Le problème n'est pas très différent de celui que posent d'autres catégories plus nobles de l'anthropologie religieuse : totémisme, fétichisme ou paganisme. On les croyait reléguées au grenier et elles reviennent malgré tout, comme si on ne pouvait ni les éradiquer complètement ni leur substituer définitivement - sans reste - tel ou tel concept savant. Ce que j'ai essayé de 
faire, après d'autres plus illustres, à propos de la catégorie de "syncrétisme » s'impose également pour la plupart des catégories du discours de l'anthropologie religieuse contemporaine: traditionalisme, prophétisme, charisme ou encore « conversion ». En un mot, la question est épistémologique autant qu'anthropologique.

Pour tous ceux qui s'efforcent de réhabiliter sinon le mot du moins la chose, la reformulation savante ou simplement métaphorique est de règle. Métissage, bricolage ou hybridité, qu'importent les métaphores ; l'essentiel est de faire comprendre que les syncrétismes religieux ne sont pas syncrétiques et encore moins syncrétistes, si l'on entend par là un mélange ou même une identification plus ou moins consciente de traditions hétérogènes, mais qu'il s'agit d'une logique de simple cumul sans confusion ou d'un processus de réinterprétation (ce que Bastide, confronté à la réalité des syncrétismes brésiliens, fut le premier à soutenir). Tout le problème vient du fait que le concept de « réinterprétation » qui, depuis Herskovits, tient lieu de "catéchisme de base " des anthropologues en matière de lecture des phénomènes de syncrétisme culturel, est un concept aveugle, une boîte noire, pire le masque des idéologies religieuses qui l'investissent. Parler de «réafricanisation » comme le font certains mouvements afro-américains ne dit rien des catégories soit-disant africaines mobilisées pour dénoncer les dérives syncrétiques issues entre autres de la contamination avec le christianisme. Comme le souligne Stefania Capone, cela sert à masquer le caractère très occidental de cette matrice de pensée qui légitime le retour à une Afrique originelle. Le concept de "réinterprétation », comme bien d'autres, doit donc être dialectisé.

La vertu du «bricolage », dans les usages que Lévi-Strauss et Bastide en ont fait, est au moins d'inciter à une réflexion sur les rapports entre métaphores et concepts et sur la construction des rapports analogiques qui président à l'élaboration des modèles d'intelligibilité des sciences sociales. Au-delà des effets de mode et des impasses d'un imaginaire condamné aux jeux de miroir, la dispute sur les métaphores (métissage ou bricolage ? syncrétisation ou créolisation ? plasticité ou hybridité ?) ne peut apporter de progrès heuristique que si les pertinences relativement circonscrites de la métaphore mobilisée permettent de poser des questions discriminantes au corpus des données d'enquête, ou si, pour reprendre une formule de Jean-Claude Passeron, « la comparaison fait disjoncter la métaphore » ${ }^{1}$.

Carmen Bernand a raison d'insister sur le fait qu'il faut contextualiser l'étude des phénomènes de syncrétisme pour mieux ajuster la comparaison, et de rappeler en premier lieu que le métissage en Amérique latine est moins une métaphore pleine d'enchantement qu'une réalité sociologique et politique. Ce que je voulais souligner à propos de la dispute sur les métaphores qu'entretiennent certains c'est le peu d'intérêt qu'il y a à investir certaines images de tous les enchantements et à charger telle ou telle autre de tous les péchés de la terre ${ }^{2}$. La réalité est que, chemin faisant, on retrouve parfois sous une métaphore les traits distinctifs qui sont liés à une autre. Selon Serge Gruzinski : "Le métissage s'exerce sur des matériaux dérivés, au sein d'une société coloniale qui se nourrit de fragments importés, de croyances tronquées, de concepts décontextualisés et souvent mal assi-

${ }^{1}$ Passeron Jean-Claude, «Analogie, Connaissance et Poésie », Revue Européenne des Sciences Sociales, 2000.

${ }^{2}$ Laplantine François, Nouss Alexis, Le métissage, Paris, Flammarion, 1997. 
milés, d'improvisations et d'ajustements pas toujours aboutis $»^{3}$. En rappelant ainsi que le métissage " aboutit à associer des motifs et des formes qui, quelle que soit leur origine, locale ou européenne, ont déjà fait l'objet d'une ou de plusieurs réinterprétations indigènes ..." ", S. Gruzinski retrouve à sa façon tout ce qui pour Lévi-Strauss fait l'intérêt de la métaphore du bricolage, à savoir l'idée d'une pré-contrainte ou d'un pré-marquage de matériaux qui ont déjà servi et qui imposent par là même certains agencements ou certains montages de sens.

Dans la rencontre et le débat qui peut se nouer entre anthropologues et sociologues à propos des productions religieuses contemporaines, cette question de la présence ou de l'effondrement des précontraintes symboliques ou culturelles qui donnent tout leur sens à la notion de travail syncrétique (ou à un syncrétisme qui travaille ...), reste à mes yeux essentielle. La conclusion de mon livre tend il est vrai, comme le souligne Frédéric Lenoir, à accentuer l'écart entre l'intérêt porté par les anthropologues aux bricolages de dispositifs essentiellement cultuels auxquels se livrent des producteurs de religions (prophètes africains ou autres) mobilisant les ressources d'une mémoire collective fragmentaire mais encore vive, et le libre bricolage d'individus consommateurs de croyances décontextualisées, de mots « désorbités », ${ }^{4}$ qui caractériserait la religion en miettes des sociétés européennes d'aujourd'hui aux yeux des sociologues ? Roger Bastide se posait déjà la question, à propos de certains mouvements noirs américains, des limites de la notion de bricolage appliquée à un contexte de déracinement culturel où les seules ressources sont celles d'une mémoire collective « en miettes ».

J'admets volontiers que l'argument que j'ai moi-même évoqué selon lequel le caractère composite et la dimension de synthèse incompatible des formations syncrétiques sont souvent un pur produit du regard du dehors, puisse en la matière m'être retourné. Rien ne remplace l'approche du bricolage « en train de se faire». En faisant crédit à certaines descriptions et analyses sociologiques du «bris-collage » postmoderne inspirées de l'héritage du "christianisme éclaté » de Michel de Certeau, j'ai conclu, sans doute un peu rapidement de cet écart, que le nouveau régime de la "religion à la carte » se nourrissait essentiellement de l'absence de " précontraintes » culturelles ou symboliques et que le caractère aléatoire et même franchement contradictoire de ses libres compositions s'apparentait plus aux configurations du kaléidoscope (autre métaphore présente chez Lévi-Strauss) qu'aux productions du bricoleur.

Mais le défi que représentent les formations syncrétiques est bien toujours le même : penser la cohérence dans l'incohérence, cerner la logique des incompatibilités. Et pour cela il faut mobiliser, comme dans l'analyse des paradoxes, une pluralité de niveaux de lecture, pratiquer la double entente. Dans la dernière version qu'elle nous livre de la disponibilité consommatrice du «bricolage croyant», Danièle Hervieu-Léger insiste sur le fait que la notion de bricolage conserve dans le nouveau régime de vérité et de légitimation du croire sa pertinence notamment parce que celui-ci n'est pas synonyme d'atomisation pure et simple des croyances individuelles : «Les individus composent librement leur " solution croyante » personnelle, mais ils le font en utilisant des ressources symboliques dont la disponibi-

\footnotetext{
${ }^{3}$ GruZinski Serge, La pensée métisse, Paris, Fayard, 1999, p. 194.

${ }^{4}$ Hervieu-LÉGer Danièle, La religion en miettes ou la question des sectes, Paris, Calmann-Lévy, 2001, p. 122.
} 
lité demeure enserrée dans certaines limites ${ }^{5}$. Parmi ces pré-contraintes qui pèsent sur la "compétence bricoleuse », le sociologue rappelle à juste titre ce que l'anthropologue tend quelque peu à gommer, à savoir le poids des dispositions sociales différenciées et du capital culturel inégal des individus consommateurs.

Mais il faut aller plus loin, et renverser effectivement le point de vue en changeant de niveau d'analyse car l'effondrement des ancrages familiaux et des héritages communautaires en matière de transmission religieuse qui n'est plus, à l'heure de la globalisation, l'apanage des sociétés européennes, a pour envers la prégnance d'un impératif culturel de la réalisation de soi qui représente, comme l'ont souligné Norbert Elias et bien d'autres, le nouveau modèle de contrôle des individus. Comme le note D. Hervieu-Léger, à propos de la figure du bouddhisme à l'occidentale étudiée par F. Lenoir, la religion humaine et libérale, accordée au modèle de la réalisation personnelle, est «une figure que le propos journalistique construit autant et plus qu'il ne la fait connaître ${ }^{6}$. L'émiettement de croyances qui faisaient système, l'affaiblissement des contraintes de la mémoire collective ou du sens des discontinuités culturelles ou historiques, facilitent le développement de religiosités délibérément syncrétistes, cosmopolites ou mêmes œcuménistes, qui libèrent la contrainte sur le contenu du croire pour mieux insister sur l'exigence de personnalisation des choix spirituels qui constitue le noyau (dur ou mou, comme on voudra) de la nouvelle matrice culturelle. Les «tyrannies de l'intimité », pour reprendre l'expression de R. Sennett, prennent le relais des contraintes communautaires ou du contrôle hiérarchique de l'institution sur le corps des croyances.

C'est évidemment à ce niveau d'analyse, non pas celui d'un syncrétisme élémentaire portant sur le contenu substantiel de telle ou telle croyance empruntée ou détournée (la réincarnation ou autres considérée isolément, comme un item), mais celui de la confrontation entre les catégories structurantes de la personne ou du mal (qui peuvent être en jeu dans l'appropriation de l'idée de réincarnation, de l'expérience de la transe ou de la figure du diable), dans ce dialogue des formes symboliques que Bastide appelait l'acculturation formelle, que la question du bricolage doit pouvoir être débattue et approfondie si l'on veut éviter les malentendus sur le «moi multiple » et sortir des pièges de l'idéologie libérale de « la religion à la carte ». Le débat n'est donc pas théologique ou théorique, le paradigme du bricolage est avant tout un outil d'analyse, plus, une invitation à se donner des contraintes d'analyse.

André MARY

Institut d'Études Africaines - MMSH

Aix-en-Provence

\footnotetext{
5 Idem, p. 127.

${ }^{6}$ Ibid, p. 127.
} 